\title{
Periungual Pyogenic Granuloma: The Importance of the Medical History
}

\author{
Aurora Alessandrini Francesca Bruni Michela Starace Bianca Maria Piraccini \\ Division of Dermatology, Department of Experimental, Diagnostic and Specialty Medicine, University of Bologna, \\ Bologna, Italy
}

\section{Established Fact}

- Pyogenic granuloma is a benign vascular tumor that can occur in different body areas, also in the nail apparatus. Treatment should be chosen according to the cause.

\section{Novel Insight}

- Patients' medical history should be more accurate in order to better recognize this tumor variant and avoid unnecessary or excessive interventions.

\section{Key Words}

Pyogenic granuloma $\cdot$ Hand eczema $\cdot$ Foreign body reaction $\cdot$ Medical history

\section{Abstract \\ Pyogenic granuloma (PG) is a common, benign vascular pro- liferation that can arise on the skin or subcutaneous tissue. It is more frequent in the early decades of life, and the most common locations are the digits of both hands and feet. The most common cause of periungual PG is drug intake, but many other trigger factors have been described in the litera- ture. Treatment should be chosen according to the cause. We describe 2 particular cases of periungual PG in which the clinical history has been fundamental. In the first case, there was an underlying hand eczema, and in the second case, a foreign body was present. \\ (c) 2016 S. Karger AG, Basel}

The authors contributed equally to this work.

\section{Introduction}

Pyogenic granuloma (PG) is a benign vascular tumor frequently observed in the first decades of life. It can occur in different body areas such as the nail apparatus, face and upper part of the chest.

PG is associated with drug intake; other important causes are local trauma, peripheral nerve injury and cast immobilization [1]. A variety of therapeutic modalities such as surgery, curettage, topical and systemic steroids, cryotherapy and laser have been used in the management of PG with variable success and recurrence rates. An effective and well-tolerated option for hand PG could be topical beta-blockers such as propranolol [2]. The choice of treatment varies depending on the cause and clinical manifestation.

We describe two types of periungual PG in which the clinical history has been essential.

\section{KARGER 125}

E-Mail karger@karger.com www.karger.com/sad
(C) 2016 S. Karger AG, Base

2296-9195/16/0014-0175\$39.50/0
Bianca Maria Piraccin

Division of Dermatology, Department of Experimental, Diagnostic and Specialty Medicine, University of Bologna

Via Massarenti, 1, IT-40138 Bologna (Italy)

E-Mail biancamaria.piraccini@unibo.it 


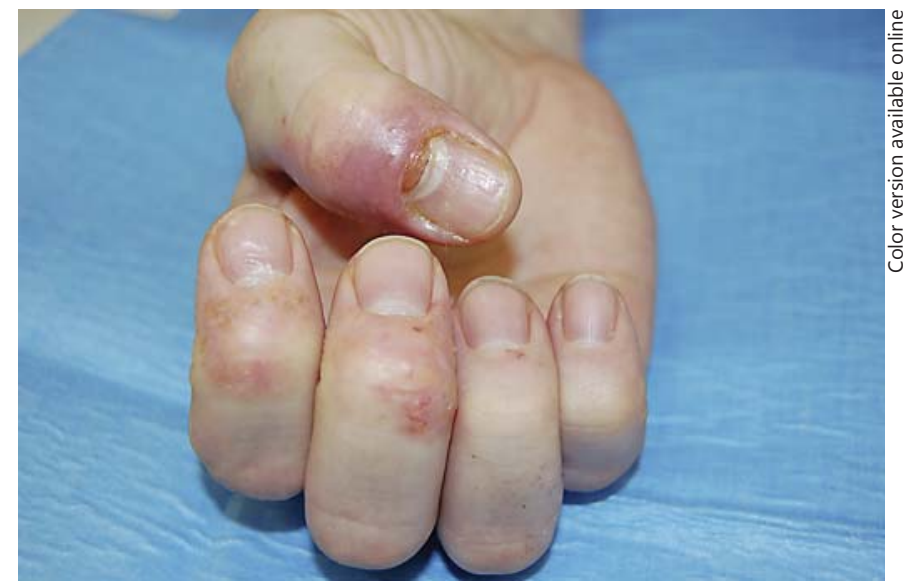

Fig. 1. PG at the proximal nail fold in a patient affected by hand eczema.

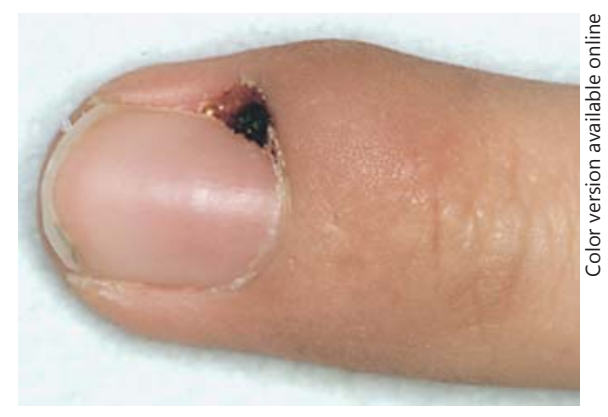

Fig. 2. PG (covered by a crust) at the lateral nail fold in a young patient due to a foreign body reaction.

\section{Case Reports}

Case 1

A 42-year-old male presented with a subacute eczema involving mainly the fingers of the right hand that had been lasting about 9 months. However, the cause of consultation was a red, painful nodule at the proximal nail fold of the first digit of the right hand, which had appeared within the previous 2 weeks. The clinical examination revealed signs of chronic hand eczema particularly marked on the distal digits, which showed skin edema and hyperkeratosis with areas of inflammation and vesicles. The cuticles of several fingernails were absent, and some nails showed Beau's lines. The first right fingernail showed the most severe changes, with severe paronychia and a bleeding nodule arising from the proximal nail fold (fig. 1). The patient denied previous trauma to the digit or intake of drugs known to induce periungual PG. He worked as a computer technician and suffered from atopic dermatitis during childhood.

Patch tests with the Italian standard series called SIDAPA (Italian Society of Allergological, Occupational and Environmental Dermatology) and some of the patient's personal products were done, and only thiomersal $0.1 \%$ caused a reaction after 48 and $72 \mathrm{~h}$. This result was considered not relevant. Positive reactions to prick tests included dates, figs, walnuts, chestnuts, hazelnuts, hon-

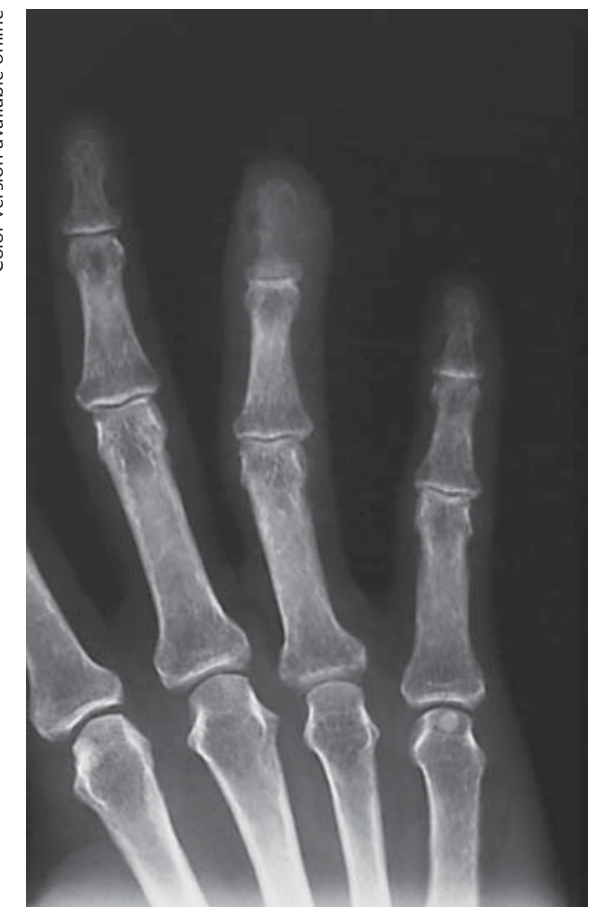

Fig. 3. X-ray showing the presence of a radio-opaque foreign body in the periungual soft tissues of the fourth finger.

ey, Dermatophagoides farinae and D. pteronyssinus, confirming an atopic diathesis.

We made a diagnosis of hand eczema due to atopic dermatitis, complicated by a periungual PG. Treatment with systemic antihistamines and topical steroids was suggested, together with surgical curettage of the PG. The pathology confirmed the clinical diagnosis of PG. At the 6-month follow-up, hand eczema was improved with cuticle regrowth, and PG had not recurred.

\section{Case 2}

We report the case of an 11-year-old Caucasian boy who presented to us with a 4-month history of a red, painful nodule at the lateral nail fold of the fourth left finger (fig. 2). The patient denied previous traumas or drug intake. He reported that he had been treated with clobetasol propionate cream under occlusion for 2 months, with partial remission of the lesion, and subsequently, he had been treated twice with diathermocoagulation. Despite these procedures, the lesion had relapsed.

Until his visit to our institution, no diagnostic imaging was recommended. Considering the age and the medical history, we immediately prescribed X-ray examination of the finger, which showed the presence of a radio-opaque, 3-mm-long, foreign body in the periungual soft tissues of the fourth finger (fig. 3). The diagnosis of PG caused by a foreign body reaction was finally made.

Further questioning revealed that the patient had had a minor bike accident with small hand abrasions 3 months ago. A surgical removal was then performed, and both the foreign body and the granulation tissue were removed. The child recovered, and PG did not relapse during the follow-up period. 


\section{Discussion}

PG, also called lobular capillary hemangioma, is a commonly acquired benign vascular lesion that can occur on the skin or subcutaneous tissue. It often appears in young individuals on the hands, nails, face and upper part of the chest.

When PG is localized in the proximal or lateral nail folds, it can involve one or more digits, presenting as a painful, smooth, sessile or pedunculated, red, rapidly growing and often bleeding lesion. PG can occur secondarily to acute or chronic local trauma, infection, drugs, i.e., retinoids, antiretrovirals, antineoplastics and pregnancy [1]. A case of periungual PG due to an anti-TNFalpha drug (etanercept) has been described by Patruno et al. [3].

Another important cause is peripheral nerve injury, frequently due to local trauma or cast immobilization [1]. In particular, mechanical trauma (e.g., manicure), friction (e.g., a prolonged walk), or an inflammatory cause (penetration of a foreign body) can predispose the development of a PG. In childhood, PGs are often self-caused because of nail biting or improper nail trimming, with penetration of spicules in the soft tissues of the nail folds and subsequent foreign body reaction, especially if the lesion is associated with digital swelling [4].

Multiple periungual PG may rarely be associated with systemic diseases such as psoriasis, cutaneous sarcoidosis, pemphigus vulgaris, Langerhans cell histiocytosis, or seronegative spondyloarthritis as a consequence of chronic paronychia [5].

The prompt diagnosis of PGs and their causes are mandatory for a correct management of such lesions. The location of PGs and the history of the patient help identify the right cause. When there is a single PG, especially if it involves the nail bed, histological examination is necessary to rule out malignant tumors such as amelanotic melanoma. When there are multiple PGs, drugs are the principle cause.

In case 1 , the medical history revealed a preexisting hand eczema, which is a common inflammatory skin disorder, affecting approximately $10 \%$ of the adult population [6]. It is probably the most common occupational skin disease. In addition, $20-35 \%$ of all types of dermatitis affect the hands [6]. Hand eczema has often a chronic course, with many implications for the patients' daily life, occupation and quality of life.

In children, atopic dermatitis is a well-documented risk factor for the development of hand eczema, which can be aggravated by irritant or allergic causes [7]. The gold standard to exclude the diagnosis of allergic contact dermatitis is patch testing, which is indicated in any patient with a chronic, pruritic, eczematous, or lichenified dermatitis [8].

Possible complications of hand eczema include bacterial or fungal superinfections, lichenification and nail disorders such as paronychia, which is frequently caused by the absence of the proximal nail fold. When hand eczema is more evident on the periungual tissue, clinical signs are represented by edema, erythema and tenderness, as in paronychia. The pathogenesis of this paronychia-induced lesion is unknown. Hand eczema induce skin fragility, including thinning of the stratum corneum and reduced keratinocyte proliferation rates, with secondary penetration of nail plate fragments into the periungual tissues.

As mentioned above, our case 1 was affected by hand eczema and subsequently developed a PG of the proximal nail fold, probably induced by inflammation due to the eczema. The nail folds were thinned and damaged by chronic inflammation, which determined a 'locus minoris resistentia' susceptible to granulomas formation.

Even though it has not been reported previously, these periungual lesions may be related to hand eczema. In our patient, they appeared at the same time, probably because the exacerbation of the hand eczema was more severe than the previous time and involved mainly the proximal nail fold.

The pathogenesis of nail PG is not completely understood. It is possible that in the periungual tissue, there is overexpression of receptors that regulate angiogenesis.

Case 2 reports a PG of the lateral nail fold of the fingernail in a young patient due to a foreign body reaction.

Foreign substances may penetrate into the skin for voluntary reasons, such as material used in tattoos, or involuntary reasons, such as accidental penetration of external substances secondary to cutaneous trauma [9]. Periungual persistent nodules and papules can be a consequence of a foreign body nail presence. Other signs, which may indicate PG, are subungual hemorrhages, chronic paronychia and symptoms such as pain and burning, with possible infection and inflammation.

Even if there is no history of traumas or drug intake, the physician has to consider an X-ray examination or a soft tissue ultrasound in order to exclude the presence of a foreign body. We always have to consider the risk of a painless penetration of little particles such as wood splinters, thin plant thorns, pencil lead, particles of tar or insect mouthparts. In fact, as it happened in our case, only after $\mathrm{X}$-ray examination and thorough questioning of the patient did he remember the minor accident. 
Furthermore, we should not forget that the correct diagnosis of most PGs is usually established by histopathological study, was not performed in our cases because of the prompt resolutions.

The purpose of this study is to underline the importance of patients' medical history, which should be more accurate in order to better recognize this tumor variant and avoid unnecessary or excessive interventions.

\section{Statement of Ethics}

Patients' consents were obtained.

\section{Disclosure Statement}

The authors do not have any conflicts of interest to declare.

\section{References}

1 Piraccini BM, Bellavista S, Misciali C, Tosti A, de Berker D, Richert B: Periungual and subungual pyogenic granuloma. $\mathrm{Br} J$ Dermatol 2010;163:941-953.

2 Piraccini BM, Alessandrini A, Dika E, Starace M, Patrizi A, Neri I: Topical propranolol 1\% cream for pyogenic granulomas of the nail: open-label study in 10 patients. J Eur Acad Dermatol Venereol 2015, Epub ahead of print.
3 Patruno C, Balato N, Cirillo T, Napolitano M, Ayala F: Periungual and subungual pyogenic granuloma following anti-TNF- $\alpha$ therapy: is it the first case? Dermatol Ther 2013;26:493495.

4 Tosti A, Piraccini BM: Pediatric diseases; in Sher RK, Daniel RC, Tosti A, Elewsky BE, Fleckman P, Rich P (eds): Nails: Diagnosis, Therapy, Surgery, ed 3. New York, Elsevier Saunders, 2005, pp 229-244.

5 Zaiac MN, Walker A: Nail abnormalities associated with systemic pathologies. Clin Dermatol 2013;31:627-649.
6 Agarwal US, Besarwal RK, Gupta R, Agarwal P, Napalia S: Hand eczema. Indian J Dermatol 2014;59:213-224.

7 Coenraads PJ: Hand eczema is common and multifactorial. J Invest Dermatol 2007;127: 1568-1570.

8 Fonacier L: A practical guide to patch testing. J Allergy Clin Immunol Pract 2015;3:669675.

9 Molina-Ruiz AM, Requena L: Foreign body granulomas. Dermatol Clin 2015;33:497-523. 